\title{
Endothelial VEGFR-3 expression in colorectal carcinomas is associated with hematogenous metastasis
}

\author{
C. JAYASINGHE, N. SIMIANTONAKI, R. MICHEL-SCHMIDT and C.J. KIRKPATRICK \\ Institute of Pathology, Johannes Gutenberg University, Mainz, Germany
}

Received April 29, 2009; Accepted June 25, 2009

DOI: $10.3892 /$ or_00000541

\begin{abstract}
Vascular endothelial growth factor receptor 3 (VEGFR-3) is a major inducer of lymphangiogenic signalling and seems to be involved also in angiogenesis. Since both processes are closely linked with tumor metastasis this study investigated the expression of VEGFR-3 in tumor-associated vessels in colorectal carcinomas and evaluated its relevance for lymphogenous and hematogenous metastasis. In a comparative study between microvascular endothelial cells isolated from the tumor (HCTEC) and the corresponding non-neoplastic tissue (HCMEC) from five patients with colorectal cancer VEGFR-3 expression was measured using a specific ELISA. The expression pattern was individually different, with cases showing reduced, elevated and unchanged protein levels. Under hypoxic culture conditions $\left(3 \% \mathrm{O}_{2}\right.$ for $24 \mathrm{~h}$ ), which are more realistic for the tumor situation, the levels remained unchanged. In contrast, hypoxia exposure of macrovascular human umbilical vein endothelial cells (HUVEC) led to a consistent downregulation of VEGFR-3 protein. These data indicate a 'hypoxia-restistant' behaviour of VEGFR-3 in colonic microvasculature. Using immunohistochemistry the endothelial expression pattern of VEGFR-3 in 74 non-metastatic, lymphogenously-metastatic and hematogenously-metastatic colorectal carcinoma specimens was assessed. Positive VEGFR-3 expression was highly significantly associated with those cases showing distant metastasis $(p=0.0003)$. In contrast, significant differences in the expression of VEGFR-3 between non-metastatic tumors and carcinomas with lymph node metastasis were not found. The majority of the detectable intratumoral VEGFR-3positive vessels were of blood vascular origin (CD31 positive, D2-40 negative). Whereas intratumoral lymphatic vessels were collapsed, VEGFR-3 positive peritumoral lymphatic
\end{abstract}

Correspondence to: Dr Caren Jayasinghe, Institute of Pathology, University Mainz, Langenbeckstrasse 1, 55101 Mainz, Germany E-mail: c.jayasinghe@gmx.de

Key words: colorectal cancer, lymphogenous metastasis, hematogenous metastasis, microvasculature, vascular endothelial growth factor receptor 3 vessels had mostly open lumina. These morphological observations provide evidence for a predominant significance of VEGFR-3-positive, possibly angiogenesis-mediated, tumor-associated blood vessels in hematogenous metastasis of colorectal cancer. In addition, due to their patency VEGFR-3positive peritumoral, but not intratumoral lymphatics could be the vascular substrate functionally mediating lymphogenous metastasis.

\section{Introduction}

The entry of neoplastic cells into lymphatic and blood vessels is the most important step for their dissemination to the regional lymph nodes and distant organs. Lymphatic and hematogenous metastasis is one of the hallmarks of malignancy and an important determinant of prognosis. The process of tumor metastasis depends mainly on the presence of an abundantly available tumoral vascular network and a successful pro-metastatic interaction between tumor cells and the vasculature $(1,2)$. Tumor angiogenesis by sprouting of new tumor blood vessels from a pre-existing vascular network promotes the development of distant metastases by augmenting the tumor-vasculature contact area and forming morphologically and functionally insufficient vessels. Hematogenous spread of cancer cells can also result via tumor cells circulating in the lymphatic vasculature, since the lymph is drained into the venous system.

Many cancer types metastasize preferentially to the regional lymph nodes via lymphatics. From an anatomical viewpoint tumor spread via lymphatic vessels is structurally and functionally easier in comparison to dissemination via blood vessels. Lymphatic vessels lack the tight interendothelial junctions as well as the surrounding layers of pericytes/smooth muscle cells and basement membranes (3). Moreover, the relatively low flow velocity and shear stress of the lymph ensure better chances of cell survival of tumor cells than in the bloodstream. The process of lymphatic metastasis is not entirely clear (4-6). So far, it is not known whether tumor invasion in pre-existing lymphatic vessels and secondary passive drainage is sufficient, or whether de novo lymphatic formation (lymphangiogenesis) is necessary for metastatic spread in lymph nodes. Additionally, the venolymphatic route is also a possible alternative pathway for lymph node metastasis via postcapillary venules by-passing lymphatic vessels. 
VEGFR-3, a member of the VEGF tyrosine kinase receptor family activated by its specific ligands VEGF-C and VEGF-D was the first lymphatic-specific growth factor receptor identified $(3,7)$. In adults VEGFR-3 is expressed mainly in lymphatic endothelial cells. However, VEGFR-3 is also expressed in quiescent endothelial cells of fenestrated microvessels of several tissue types. Under pathological conditions, especially in the case of active angiogenesis in tumor-associated endothelium, VEGFR-3 is transiently induced. VEGFR-3 is a major inducer of lymphangiogenic signalling by preventing lymphatic endothelium apoptosis and stimulating migration, proliferation and cell survival. In addition to lymphangiogenesis, VEGFR-3 seems to be involved in (hem)angiogenesis by maintaining the integrity of the new-formed endothelial cell lining and controlling angiogenic sprouting and vascular network formation $(8,9)$.

Although experimental studies have indicated that VEGFR-3 plays an important role in (lymph)angiogenesis the clinical significance remains unclear. Particularly in the case of colorectal cancer, VEGFR-3 expression has already been recognised in both lymphatics and blood vessels (10-14). However, a clear association between endothelial VEGFR-3 expression and the metastatic disease status has not been described so far.

In order to find out whether VEGFR-3 is relevant for the metastatic behavior of colorectal cancer we examined systematically its endothelial expression. Firstly we determined whether VEGFR-3 expression in the tumor-associated vessels differs from the endothelium in non-neoplastic tissue. In this context, we compared in an in vitro study the expression of VEGFR-3 in human colonic tumor-derived microvascular endothelial cells (HCTEC) and human colonic microvascular endothelial cells (HCMEC) from the corresponding non-neoplastic tissue isolated from several patients with colorectal cancer under normoxic, and for the tumor situation more realistic, hypoxic culture conditions. Moreover, the VEGFR-3 expression patterns in colonic microvasculature were compared with the expression profiles in macrovascular human umbilical vein endothelial cells (HUVEC) of different donors. In the next step we investigated the endothelial expression pattern of VEGFR-3 in a series of 74 non-metastatic, lymphatic-metastatic and hematogenousmetastatic colorectal carcinomas using immunohistochemistry. Additionally, we evaluated in situ whether VEGFR-3 expressing tumor vessels were lymphatic or blood cells.

\section{Materials and methods}

Isolation and culture of HUVEC. HUVEC were isolated from human umbilical cords and cultured as previously described (15). For all experiments HUVEC in passage 2 were used.

Isolation and culture of HCMEC and HCTEC. HCTEC and HCMEC were isolated from the tumor and the corresponding normal colonic tissue of the same donor and cultured as previously described (16). For all experiments endothelial cells in passage 3 were used.

Immunofluorescence. HCTEC and HCMEC were seeded onto fibronectin-coated glass chamber-slides (LabTek, Nunc, Wiesbaden, Germany). After growing to subconfluence cells were fixed with buffered $3.7 \%$ paraformaldehyde (15 min, room temperature) and permeabilized with $0.1 \%$ Triton X-100 (5 min, room temperature). Rabbit polyclonal anti-VEGFR-3 (Chemicon, Düsseldorf, Germany) was used as primary antibody. Alexa 546 goat anti-rabbit (Eugene, OR, USA) was used as secondary antibody. Nuclear staining was performed with Hoechst 33342 (Sigma, Taufkirchen, Germany). Fluorescently labeled cells were covered with GelMount.

ELISA analysis. Levels of VEGFR-3 were measured in cell lysates of HCMEC and HCTEC of 5 patients cultivated under normoxic $\left(21 \% \mathrm{O}_{2}\right)$ and hypoxic $\left(3 \% \mathrm{O}_{2}\right)$ conditions over $24 \mathrm{~h}$. For the quantitative determination of VEGFR-3 concentrations in cell lysates commercially available sandwich ELISA assays (R\&D systems, Wiesbaden, Germany) were used according to the manufacturer's instructions. Cell lysates were prepared using a lysis buffer provided with the kit. The optic density of the color reaction was determined using a microplate ELISA reader set to $450 \mathrm{~nm}$.

Tissue samples. The colorectal tissue samples used in this study came from 74 patients undergoing elective surgery for colorectal cancer at the University of Mainz during the years 1995-1999. The investigation of these tissues was in accordance with the rules of the responsible state ethics committee of the Mainz University. The morphological classification of the carcinomas was conducted according to WHO specifications (18). All tumors were staged following the guidelines of the TNM Classification of Malignant Tumors. With respect to the $\mathrm{T}$ status all tumors investigated were $\mathrm{T} 3$ and moderately differentiated (G2), and were separated into three groups according to metastatic status. The first group comprised 30 cases without tumor metastasis to regional lymph nodes or distant organs (N0/M0). Among the remaining 44 metastasizing cases, 20 were characterized by lymphogenous $(\mathrm{N}+)$ and 24 by haematogenous metastases $(\mathrm{M}+)$. For all samples investigated, follow-up data were obtained from hospital charts and by corresponding with the physicians in charge during a period of 5 years after surgery.

Immunohistochemistry. All immunohistochemical reactions were conducted using formalin-fixed and paraffin-embedded samples. After deparaffination the samples were treated in a microwave oven in EDTA buffer for $15 \mathrm{~min}$. Incubation with the primary antibodies rabbit polyclonal flt-4/VEGFR-3 (C-20; Santa Cruz Biotechnology, Inc., Santa Cruz, USA), mouse monoclonal CD31 (Dako, Hamburg, Germany), mouse monoclonal D2-40 (Signet, Dedham, MA, USA) and the secondary antibodies, horse anti-mouse biotinylated IgG (for CD31 and D2-40, Vector; Burlingame, CA, USA) and rabbit anti-goat biotinylated IgG (for VEGFR-3, Vector) were carried out in accordance with standard protocols using the Vectastain Elite reagent (Vector). All primary antibodies were used at a dilution of 1:100. All secondary antibodies employed in this study were used at a dilution of 1:200. Sections were counterstained with Mayer's hematoxylin. To prove the specificity of the immunoreactions, a sample from every colorectal carcinoma sample $(n=74)$ was stained solely with the secondary antibody, omiting the primary antibody as negative control. Immunostaining reactions of each sample were evaluated by two authors independently (N.S. and C.J) 


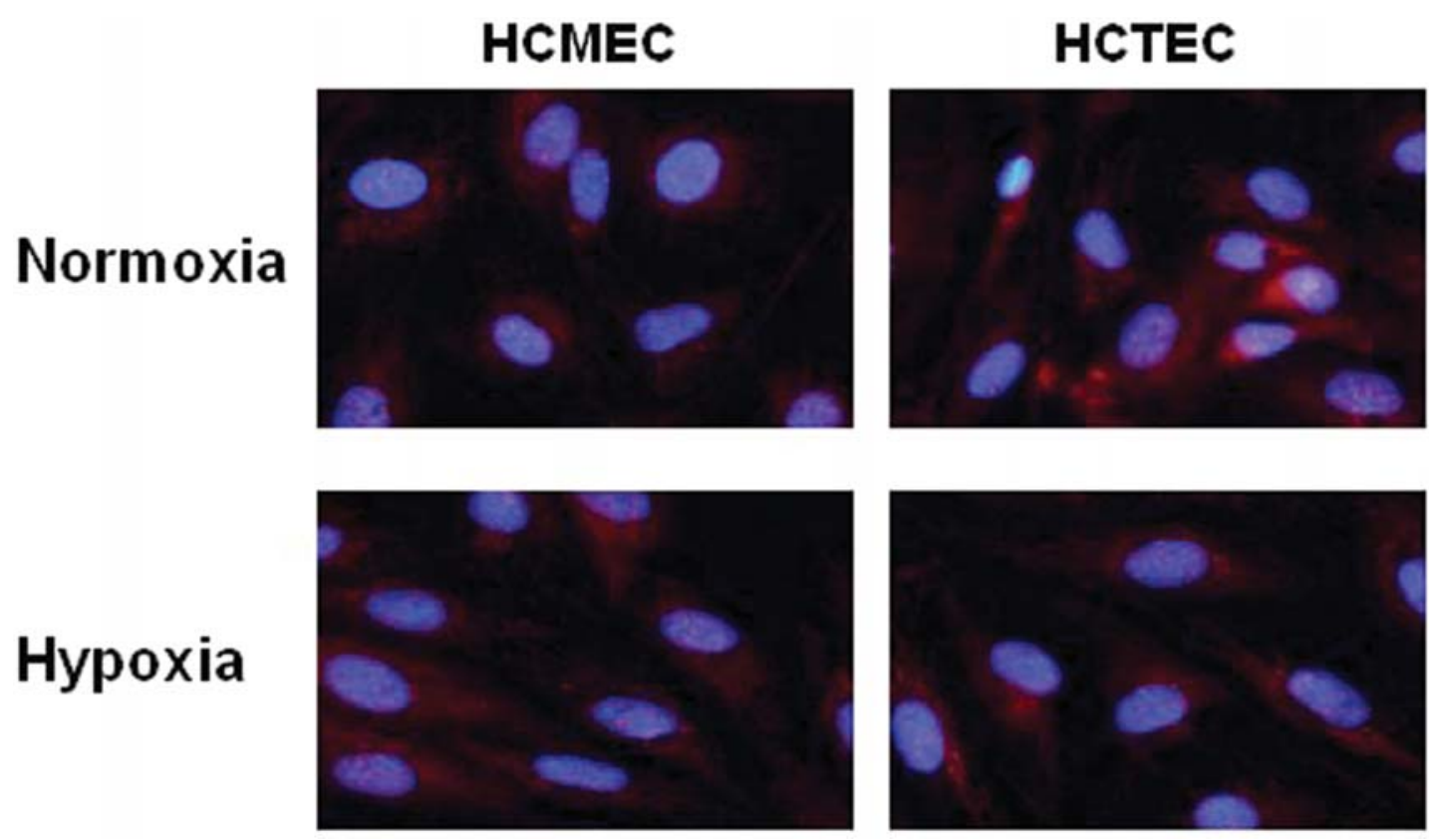

Figure 1. Immunofluorescence for VEGFR-3 expression in HCMEC and corresponding HCTEC. VEGFR-3 protein is detected in both under normoxia $\left(21 \% \mathrm{O}_{2}\right)$ and hypoxia $\left(3 \% \mathrm{O}_{2}\right)$

without knowledge of the clinical data. The intensity of endothelial VEGFR-3 staining was scored on a semi-quantitative scale from 0 to 2 ( 0 , no staining; 1 , weak staining and 2 , strong staining). D2-40 and CD31 staining was either negative or positive.

Statistical analysis. Statistical analyses of the ELISA analysis were performed using the Wilcoxon signed rank test. Significance was set at a $p<0.05$. The evaluation of data concerning association of immunostaining reaction with tumor stage was assessed using $\chi^{2}$ (Fisher's exact test). $\mathrm{p}<0.05$ was considered to be significant in all statistical analyses.

\section{Results}

VEGFR-3 is differentially expressed in HCMEC and HCTEC. For the following comparative studies we isolated from the non-neoplastic and neoplastic colon tissue pairwise the normal microvasculature (HCMEC) and the corresponding tumor-associated microvasculature (HCTEC). Using immunofluorescence expression of VEGFR-3 protein under normoxia and hypoxia was found in both endothelial types (Fig. 1). As shown in Fig. 2 marked change in the VEGFR-3 levels could not be found after $24 \mathrm{~h}$ of hypoxia either in HCMEC or in HCTEC. The mean value of VEGFR-3 levels was 57 and $53 \mathrm{pg} / \mathrm{ml}$ protein in HCMEC and 60 and $57 \mathrm{mg} / \mathrm{ml}$ protein in HCTEC under normoxia and hypoxia, respectively. The normoxic and hypoxic VEGFR-3 expression pattern of HCTEC and corresponding HCMEC was variable and individually different. VEGFR-3 was significantly elevated in two cases (patient 1 and patient 3 ) and reduced in one case (patient 2) in HTMEC in comparison to the corresponding HCMEC under both conditions of normoxia and hypoxia. HCMEC and HCTEC of two patients (patiens 4 and patient 5) exhibited the same VEGFR-3 concentrations under
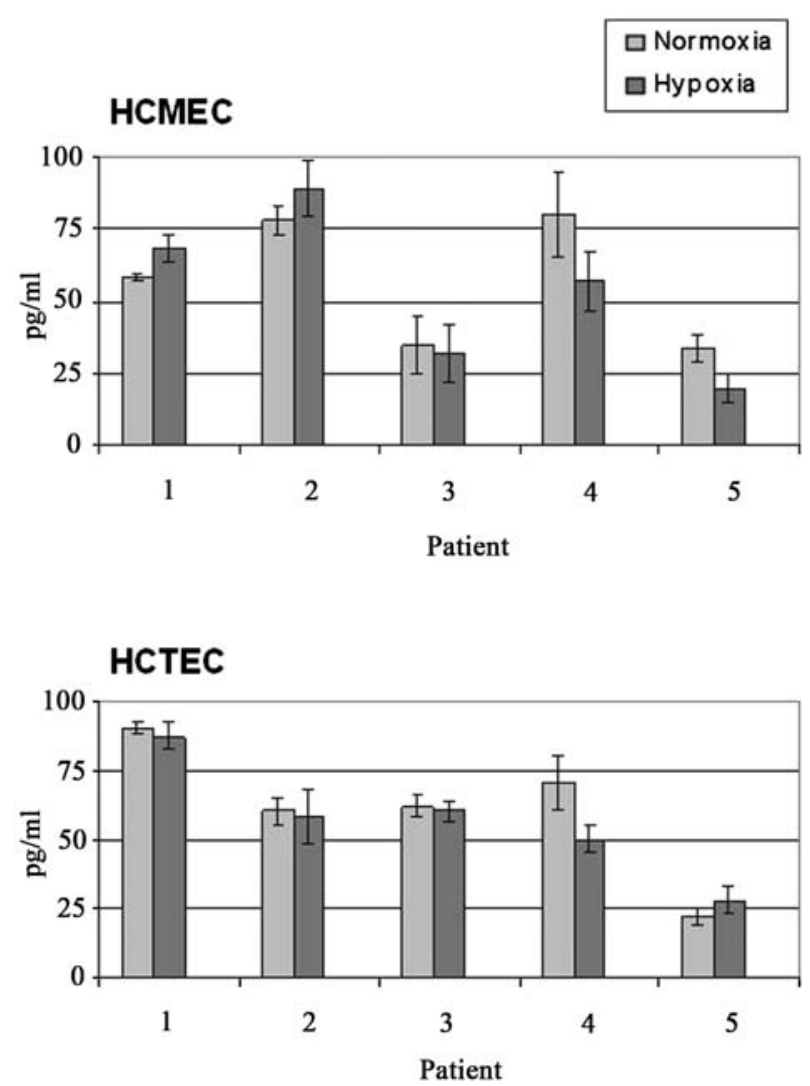

Figure 2. Determination of VEGFR-3 concentrations by ELISA in HCMEC and corresponding HCTEC from five patients. Comparison of normoxic conditions $\left(21 \% \mathrm{O}_{2}\right)$ and after exposure to hypoxia $\left(3 \% \mathrm{O}_{2}\right)(\mathrm{n}=3$, mean values \pm standard error).

both oxygen conditions. VEGFR-3 level changes of HCTEC and the corresponding HCMEC of the same patient were unidirectional under normoxia and hypoxia in all cases. 
A
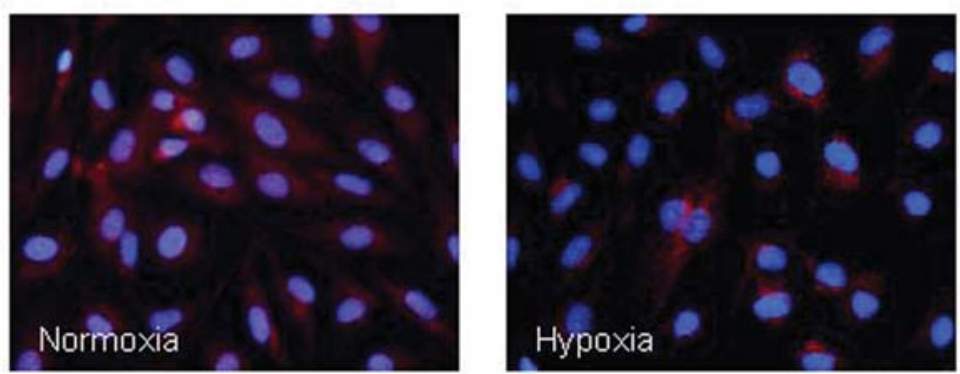

B

\section{HUVEC}

$\square$ Normoxia

口 Hypoxia

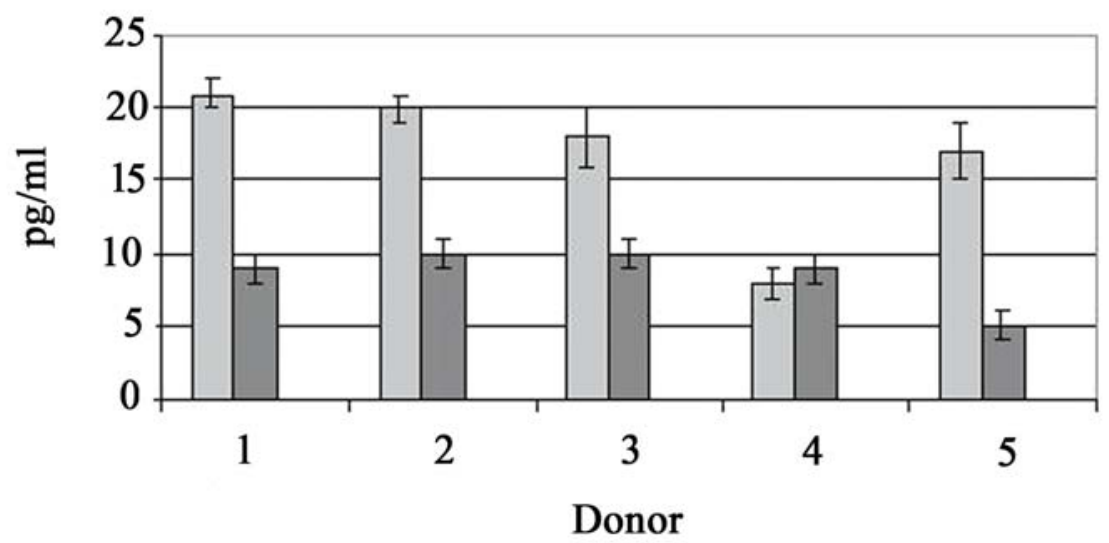

Figure 3. VEGFR-3 expression in HUVEC under normoxia $\left(21 \% \mathrm{O}_{2}\right)$ and hypoxia $\left(3 \% \mathrm{O}_{2}\right)$. (A) Using immunofluorescence VEGFR-3 protein is detected in HUVEC under both oxygen conditions. (B) Comparative analysis of VEGFR-3 concentrations by ELISA in HUVEC from five donors ( $\mathrm{n}=3$, mean values \pm standard error).

VEGFR-3 expression is reduced in HUVEC under hypoxic conditions. To determine whether VEGFR-3 is differently expressed on HUVEC in comparison to the colonic microvasculature their protein expression was examined under normoxic $\left(21 \% \mathrm{O}_{2}\right)$ and hypoxic conditions $\left(3 \% \mathrm{O}_{2}\right)$ using immunofluorescence. As shown in Fig. 3A for both oxygen concentrations VEGFR-3 was expressed in HUVEC. As next step, we examined the concentrations of VEGFR-3 in HUVEC of five different donors and compared their amounts under normoxia and hypoxia using a specific ELISA. Under hypoxic conditions a uniform significant decrease of VEGFR-3 in HUVEC was observed in four cases (range $17-21 \mathrm{pg} / \mathrm{ml}$ protein under normoxia vs. $5-10 \mathrm{pg} / \mathrm{ml}$ under hypoxia; p<0.001) (Fig. 3B). It is noteworthy that under both conditions HUVEC exhibited significantly lower levels of VEGFR-3 in comparison to HCMEC and HCTEC.

Endothelial VEGFR-3 is overexpressed in hematogenousmetastatic colorectal carcinomas. To elucidate the relevance of VEGFR-3 for colorectal cancer metastasis, immunohistochemistry was used to determine its endothelial expression in 30 non-metastatic (N0/M0) and 20 lymphogenouslymetastatic $(\mathrm{N}+)$, as well as 24 hematogenously-metastatic $(\mathrm{M}+)$ colorectal carcinomas. For the best possible statistical
Table I. Distribution of non-metastatic (N0/M0), lymphogenous-metastatic $(\mathrm{N}+)$ and hematogenous-metastatic $(\mathrm{M}+)$ colorectal carcinomas according to endothelial VEGFR-3 expression of tumor-associated vessels.

\begin{tabular}{lcccc}
\hline Colorectal carcinomas & No. & Negative & Low & High \\
\hline N0/M0 & 30 & 20 & 3 & 7 \\
N+ & 20 & 10 & 6 & 4 \\
M+ & 24 & 4 & 5 & 15 \\
\hline
\end{tabular}

No., number of cases examined.

comparison all tumors investigated were with respect to the $\mathrm{T}$ status and grading, defined as T3 (subserosa infiltration) and moderately differentiated (G2).

Positive immunohistochemical expression of VEGFR-3 was observed in the membrane and cytoplasm of the intratumoral endothelial cells in 33,50 and $83 \%$ of the nonmetastatic, lymphogenously-metastatic and hematogenouslymetastatic cases, respectively (Table I). Since endothelial VEGFR-3 staining intensity was variable, tumors were 

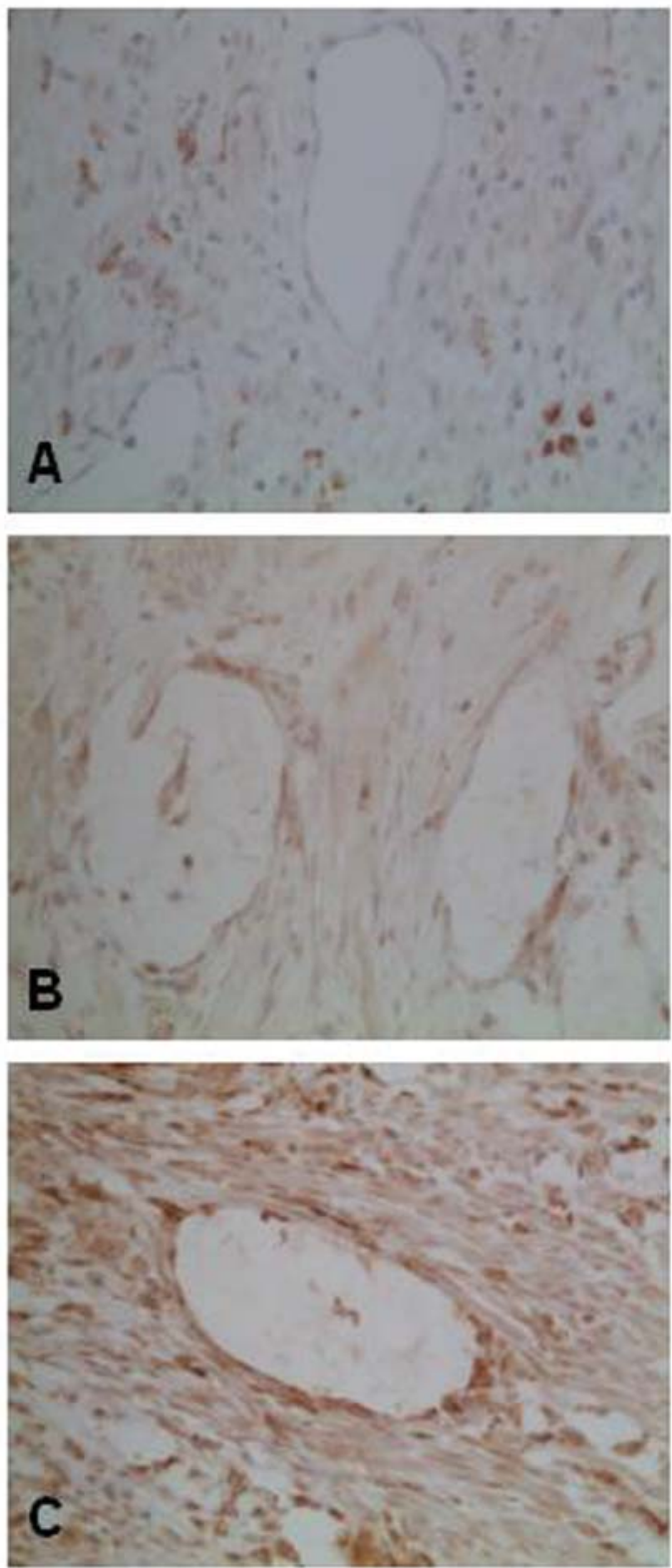

Figure 4. Endothelial VEGFR-3 expression profiles detected in colorectal cancer. (A) Tumor-associated vessels negative for VEGFR-3. (B) Vessels characterized by a weak stain. (C) Vessel with endothelial cells showing a strong immunoreactivity. Magnification, x400.

classified into three groups, namely with negative, low and high expression (Fig. 4). The topological staining distribution of the endothelial VEGFR-3 expession was homogeneous. No differences of staining intensity and density between the superficial tumor fraction and the invasive tumor edge were observed. Positive VEGFR-3 expression was significantly associated with distant metastasic status. This was the case in both the comparison between non-metastatic and hematogenously-metastatic tumors $(\mathrm{p}=0.0003)$ as well as between lymphogenously-metastatic and hematogenously-metastatic tumors $(\mathrm{p}=0.025)$. There were no identifiable significant differences in the expression of VEGFR-3 between nonmetastatic tumors and carcinomas with lymph node metastasis.

The VEGFR-3 positive vessels in colorectal cancer are mainly of blood vascular origin. In the next step we determined
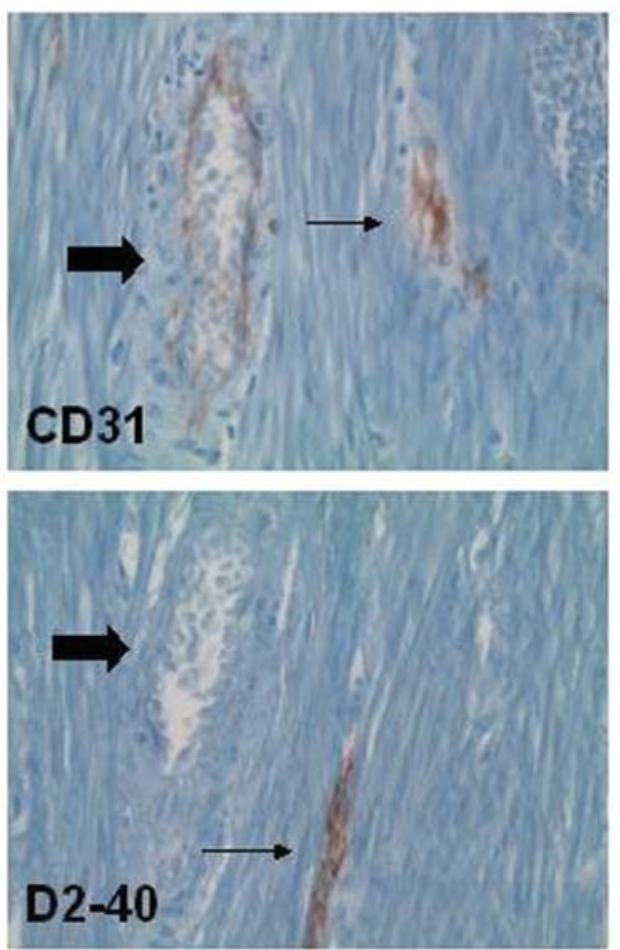

Figure 5. CD31 and D2-40 immunoreactivity in tumor-associated vessels in colorectal cancer. Anti-CD31 antibody stained both blood (thick arrow) and lymphatic (thin arrow) vessels. Lymphatic vessels stained positively for D2-40, whereas blood vessels showed an absent immunoreactivity. Magnification, $\mathrm{x} 400$.

whether the VEGFR-3-positive vessels within the tumor stroma were blood or lymphatic vessels. To distinguish these two types of vessels, we stained serial sections with the specific lymphatic endothelial marker D2-40 and the general endothelial marker CD31 (Fig. 5). The majority of the VEGFR-3-positive vessels (about 80-90\%) were strongly positive for CD31 but negative for D2-40, therefore identifying them as blood vessels. These vessels often contained erythrocytes, confirming that they were in fact of blood vascular origin (Fig. 6A). A great number of the D2-40-positive lymphatic vessels were strongly compressed from the surrounding stroma and were recognized conspicuously only in the immunohistochemically stained sections (Fig. 6B). Interestingly, lymphatic vessels with open lumina were seen mostly in the tumor surface and peritumorally directly beneath the deep infiltration front (Fig. 6C).

In the non-neoplastic tissue VEGFR-3 expression was detected in lymphatic vessels and blood microvessels (Fig. 7). Both vascular types were found in the submucosa from which HCMEC were isolated. Thus, the isolated VEGFR-3 positive endothelia of both the tumor (HCTEC) and the non-neoplastic tissue (HCMEC) were a mixture of endothelial cells of blood and lymphatic origin.

\section{Discussion}

The anatomical relation and functional interaction between malignant tumor and lymphatic as well as blood vasculature are important factors determining lymphatic and distant metastasis. In the present analysis we focused on investigating 

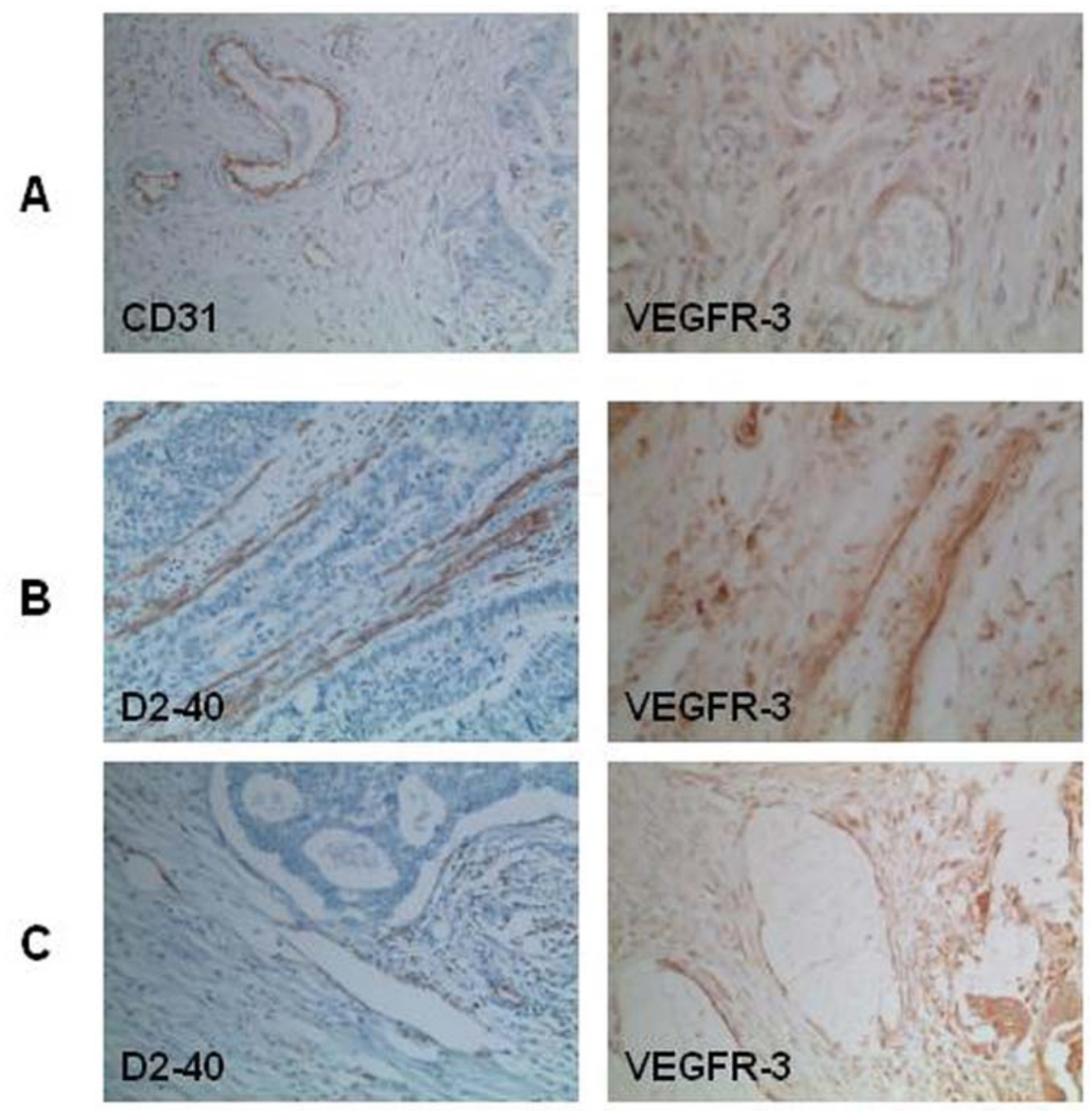

Figure 6. Morphology of VEGFR-3 positive blood (A) as well as intratumoral (B) and peritumoral (C) lymphatic vessels. (A) CD31-positive blood vessels had open lumina filled with erythrocytes. (B) D2-40 positive intratumoral lymphatics were collapsed. (C) D2-40 positive peritumoral lymphatics had open and in part dilatated lumina.

the possible relevance of endothelial VEGFR-3 expression for the process of colorectal cancer metastasis. Using a specific ELISA we carried out a comparative study between tumorderived endothelium (HCTEC) and the corresponding nonneoplastic colonic endothelium (HCMEC) from five patients separately and found that the expression manner was individually different, with cases with reduced, elevated and unchanged VEGFR-3 levels. The measure of similar VEGFR-3 concentrations in the tumor-associated and normal endothelial cells in two cases could reflect an initially equal basal VEGFR-3 expression in the normal and tumor tissue. Since VEGFR-3 is suggested to be expressed in pre-existing, but also in newly formed tumor blood and lymphatic vasculature upregulation of VEGFR-3 in tumor tissue could mean increased (lymph)angiogenic capacity and downregulation of VEGFR-3 could imply decreased (lymph)angiogenesis as a protective activity. Interestingly, VEGFR-3 levels remained unchanged after exposure to hypoxia in HCMEC and HCTEC. Moreover, VEGFR-3 level changes of HCTEC and the corresponding HCMEC of the same patient were unidirectional under normoxia and hypoxia in all cases. These observations indicate a 'hypoxia-restistant' behavior of VEGFR-3 in colonic microvasculature. In contrast, hypoxia exposure of HUVEC, the most commonly used endothelial culture type, caused consistent down-regulation of the VEGFR-3 protein. Thus, intrinsic cell-specific differences exist between microvascular and macrovascular endothelial cells in response to hypoxia by unknown regulatory mechanisms. In agreement with our results Aparicio et al found reduced VEGFR-3 transcript levels in HUVEC under hypoxia (18). In comparison to HUVEC, HCMEC/HCTEC expressed high levels of VEGFR-3 protein an observation that suggests a particular role of VEGFR-3 for the colonic microvasculature.

The inconsistent VEGFR-3 expression pattern was also reflected in the immunohistochemical VEGFR-3 analysis in colorectal carcinomas in situ with cases having negative, low and high endothelial expression. These data offer a very sensitive regulation system, which becomes more complicated by additive paracrine stimulation of the endothelium in vivo. In our study positive endothelial VEGFR-3 expression was not correlated with lymph node metastasis. In concordance with our results, White et al did not find a significant correlation between VEGFR-3 positive endothelial cell density and nodal metastases (13). In non-small cell lung cancer VEGFR-3 positive vessel density was not correlated with nodal positive cases (19). However, in another study with lung adenocarcinomas as well as in prostate and breast cancer 

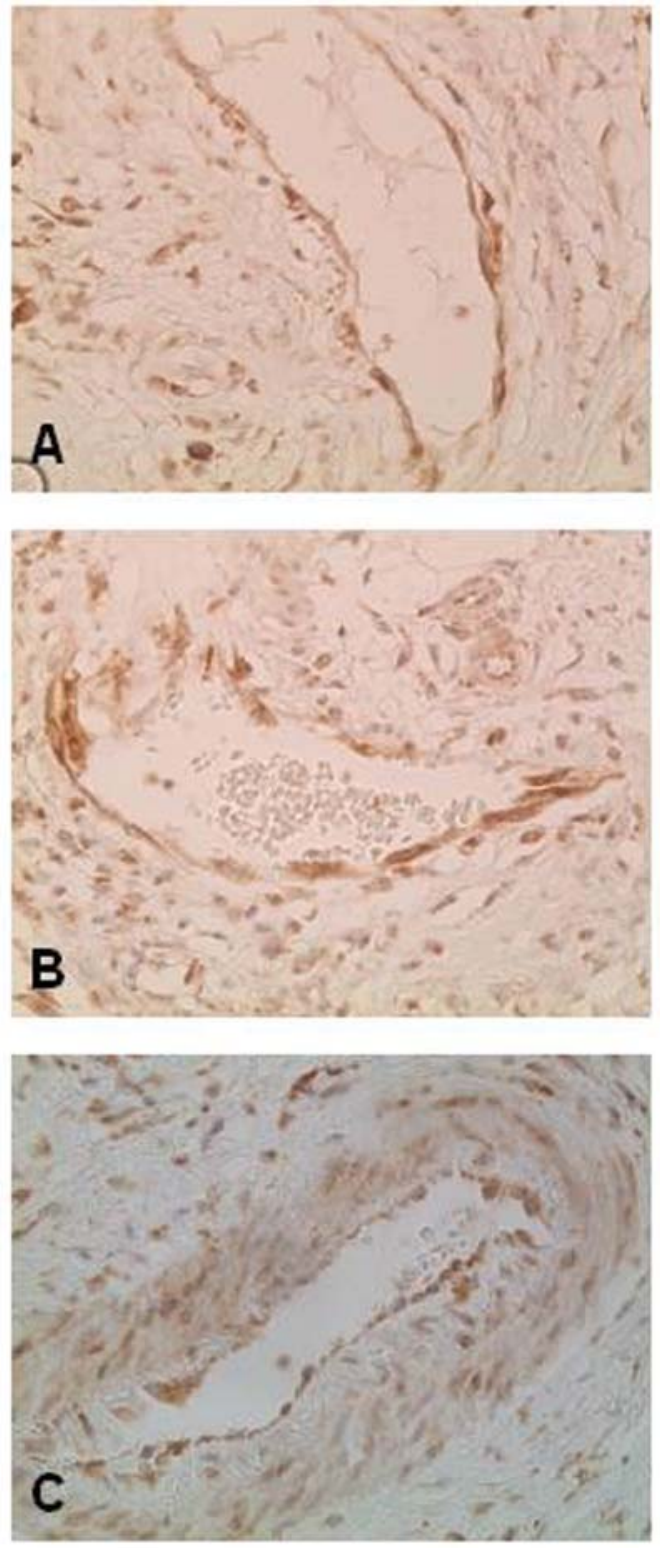

Figure 7. VEGFR-3 positive vessels in non-neoplastic colonic tissue. Positive endothelial VEGFR-3 expression was detected in lymphatic vessels (A) and blood microvessels, especially in venules (B) and arterioles (C).

endothelial VEGFR-3 expression was described as being associated with lymph node metastasis (20-22). The exact reason for these inconsistences is unclear. An autocrine and/or paracrine pathway possibly including both VEGFR-3 ligands, VEGF-C and VEGF-D, could act as an interactive communication between the components of a dynamic system and thus determine the potential for lymph node metastasis. Using the specific lymphatic endothelium marker D2-40 only a minority of intratumoral vessels were lymphatic. Lack of lumen formation was a common feature of intratumoral lymphatics, whereas lymphatic vessels with open lumina were seen mostly at the tumor surface and peritumorally directly beneath the deep infiltration front. This morphological distribution suggests a predominant role of peritumoral lymphatics in lymphogenous metastasis, because collapsed intratumoral lymphatic are not suitable for tumor cell dissemination. Collapsed intratumoral lymphatics could reflect a mechanical stress situation induced by the vessel pressure in the desmoplastic tumor tissue. By a compensatory mechanism the resulting elevated lymph pressure could lead to a dilatation of the lymphatics in the tumor periphery. Recent evidence indicates that the distinction between intratumoral lymphatic vessels and peritumoral lymphatics is meaningful for the study of lymphatic metastasis. However, the functional significance of both lymphatic vessel subtypes is controversially discussed. Whilst, as in the present study, it has been proposed that intratumoral lymphatic vessels can not transport tumor cells other studies have reported intratumoral lymphatic density as an important indicator for metastatic propensity (23-28).

Surprisingly, in the present immunohistochemical analysis VEGFR-3 expression in tumor-associated vessels was correlated significantly with colorectal carcinomas demonstrating distant metastasis. To our knowledge studies on a positive correlation between endothelial VEGFR-3 expression and distant metastasis of carcinomas, including colorectal carcinomas, do not yet exist. Tumor metastasis in distant organs is mostly hematogenous in nature via the systemic circulation and is closely associated with angiogenesis $(1,2)$. Angiogenic vessels are leaky with fenestrated endothelium and increased microvascular permeability, factors that promote tumor cell penetration. Since the majority of the detectable intratumoral VEGFR-3-positive vessels were of blood vascular origin we suppose that distant metastasis is closely linked with tumor-induced angiogenesis.

This assumption is supported by previous experimental studies which indicated that VEGFR-3 is expressed in blood vessels of tumors and wounds undergoing angiogenesis $(29,30)$. In a recently published article it has been demonstrated that VEGFR-3 is highly expressed in angiogenic sprouts and blocking of VEGFR-3 signalling resulted in inhibition of angiogenesis (9). In contrast to the compressed lymphatics within the tumor, the lumina of the intratumoral blood vessels were open. A possible explanation could be the relative high intravasal pressure in the blood stream in comparison to the lymph.

In conclusion, this study provides first insights into the significance of VEGFR-3-positive, possibly angiogenesismediated, tumor-associated blood vessels in hematogenous metatastasis of colorectal cancer. VEGFR-3-positive peritumoral lymphatics could represent a vascular substrate for signaling network interactions mediating lymphogenous metastasis.

\section{References}

1. Kopfstein L and Christofori G: Metastasis: cell-autonomous mechanisms versus contributions by the tumor microenvironment. Cell Mol Life Sci 63: 449-468, 2006.

2. Langley RR and Fidler IJ: Tumor cell-organ microenvironment interactions in the pathogenesis of cancer metastasis. Endocr Rev 28: 297-321, 2007.

3. Alitalo K and Carmeliet P: Molecular mechanisms of lymphangiogenesis in health and disease. Cancer Cell 1: 219-227, 2002.

4. Shayan R, Achen MG and Stacker SA: Lymphatic vessels in cancer metastasis: bridging the gaps. Carcinogenesis 27: 1729-1738, 2006.

5. Tobler NE and Detmar M: Tumor and lymph node lymphangiogenesis - impact on cancer metastasis. J Leukoc Biol 80: 691-696, 2006. 
6. Wong SY and Hynes RO: Lymphatic or hematogenous dissemination: how does a metastatic tumor cell decide? Cell Cycle 5: 812-817, 2006

7. Kaipainen A, Korhonen J, Mustonen T, van Hinsbergh VW, Fang GH, Dumont D, Breitman M and Alitalo K: Expression of the fms-like tyrosine kinase 4 gene becomes restricted to lymphatic endothelium during development. Proc Natl Acad Sci USA 92: 3566-3570, 1995.

8. Kubo H, Fujiwara T, Jussila L, Hashi H, Ogawa M, Shimizu K, Awane M, Sakai Y, Takabayashi A, Alitalo K, Yamaoka Y and Nishikawa SI: Involvement of vascular endothelial growth factor receptor-3 in maintenance of integrity of endothelial cell lining during tumor angiogenesis. Blood 96: 546-553, 2000.

9. Tammela T, Zarkada G, Wallgard E, Murtomäki A, Suchting S, Wirzenius M, Waltari M, Hellström M, Schomber T, Peltonen R, Freitas C, Duarte A, Isoniemi H, Laakkonen P, Christofori G, Ylä-Herttuala S, Shibuya M, Pytowski B, Eichmann A, Betsholtz C and Alitalo K: Blocking VEGFR-3 suppresses angiogenic sprouting and vascular network formation. Nature 454: 656-660, 2008.

10. George ML, Tutton MG, Janssen F, Arnaout A, Abulafi AM, Eccles SA and Swift RI: VEGF-A, VEGF-C and VEGF-D in colorectal cancer progression. Neoplasia 3: 420-427, 2001.

11. Witmer AN, van Blijswijk BC, Dai J, Hofman P, Partanen TA, Vrensen GF and Schlingemann RO: VEGFR-3 in adult angiogenesis. J Pathol 195: 490-497, 2001.

12. Clarijs R, Schalkwijk L, Hofmann UB, Ruiter DJ and de Waal RM: Induction of vascular endothelial growth factor receptor-3 expression on tumor microvasculature as a new progression marker in human cutaneous melanoma. Cancer Res 62: 7059-7065, 2002.

13. White JD, Hewett PW, Kosuge D, McCulloch T, Enholm BC, Carmichael J and Murray JC: Vascular endothelial growth factor-D expression is an independent prognostic marker for survival in colorectal carcinoma. Cancer Res 62: 1669-1675, 2002 .

14. Onogawa S, Kitadai Y, Tanaka S, Kuwai T, Kuroda T and Chayama K: Regulation of vascular endothelial growth factor (VEGF)-C and VEGF-D expression by the organ microenvironment in human colon carcinoma. Eur J Cancer 40: 1604-1609, 2004.

15. Simiantonaki N, Jayasinghe $\mathrm{C}$ and Kirkpatrick CJ: Effect of pro-inflammatory stimuli on tumor cell-mediated induction of endothelial cell adhesion molecules in vitro. Exp Mol Pathol 73 46-53, 2002

16. Jayasinghe C, Simiantonaki N, Michel-Schmidt R and Kirkpatrick CJ: Comparative study of human colonic tumorderived endothelial cells (HCTEC) andnormal colonic microvascular endothelial cells (HCMEC): Hypoxia-induced sVEGFR-1 and sVEGFR-2 levels. Oncol Rep 21: 933-939, 2009.

17. Sobin LH and Wittekind C (ed): International Union Against Cancer (UICC). TNM Classification of Malignant Tumors, fifth edition, 1997

18. Aparicio S, Sawant S, Lara N, Barnstable CJ and Tombran-Tink J: Expression of angiogenesis factors in human umbilical vein endothelial cells and their regulation by PEDF. Biochem Biophys Res Commun 326: 387-394, 2005.
19. Chen F, Takenaka K, Ogawa E, Yanagihara K, Otake Y, Wada H and Tanaka F: Flt-4-positive endothelial cell density and its clinical significance in non-small cell lung cancer. Clin Cancer Res 10: 8548-8553, 2004.

20. Niki T, Iba S, Yamada T, Matsuno Y, Enholm B and Hirohashi S: Expression of vascular endothelial growth factor receptor 3 in blood and lymphatic vessels of lung adenocarcinoma. J Pathol 193: 450-457, 2001.

21. Zeng Y, Opeskin K, Baldwin ME, Horvath LG, Achen MG, Stacker SA, Sutherland RL and Williams ED: Expression of vascular endothelial growth factor receptor-3 by lymphatic endothelial cells is associated with lymph node metastasis in prostate cancer. Clin Cancer Res 10: 5137-5144, 2004.

22. Nakamura $Y$, Yasuoka H, Tsujimoto M, Yang Q, Imabun S, Nakahara M, Nakao K, Nakamura M, Mori I and Kakudo K: Flt-4-positive vessel density correlates with vascular endothelial growth factor-d expression, nodal status, and prognosis in breast cancer. Clin Cancer Res 9: 5313-5317, 2003.

23. Padera TP, Kadambi A, di Tomaso E, Carreira CM, Brown EB, Boucher Y, Choi NC, Mathisen D, Wain J, Mark EJ, Munn LL and Jain RK: Lymphatic metastasis in the absence of functional intratumor lymphatics. Science 296: 1883-1886, 2002.

24. Leu AJ, Berk DA, Lymboussaki A, Alitalo K and Jain RK: Absence of functional lymphatics within a murine sarcoma: a molecular and functional evaluation. Cancer Res 60: 4324-4327, 2000.

25. Yokoyama Y, Charnock-Jones DS, Licence D, Yanaihara A, Hastings JM, Holland CM, Emoto M, Sakamoto A, Sakamoto T, Maruyama H, Sato S, Mizunuma H and Smith SK: Expression of vascular endothelial growth factor (VEGF)-D and its receptor, VEGF receptor 3, as a prognostic factor in endometrial carcinoma. Clin Cancer Res 9: 1361-1369, 2003.

26. Yokoyama Y, Charnock-Jones DS, Licence D, Yanaihara A, Hastings JM, Holland CM, Emoto M, Umemoto M, Sakamoto T, Sato S, Mizunuma H and Smith SK: Vascular endothelial growth factor-D is an independent prognostic factor in epithelial ovarian carcinoma. Br J Cancer 88: 237-244, 2003.

27. Zeng Y, Opeskin K, Horvath LG, Sutherland RL and Williams ED: Lymphatic vessel density and lymph node metastasis in prostate cancer. Prostate 65: 222-230, 2005.

28. Schoppmann SF, Bayer G, Aumayr K, Taucher S, Geleff S, Rudas M, Kubista E, Hausmaninger H, Samonigg H, Gnant M, Jakesz R and Horvat R: Austrian breast and colorectal cancer study group. Prognostic value of lymphangiogenesis and lymphovascular invasion in invasive breast cancer. Ann Surg 240: 306-312, 2004.

29. Valtola R, Salven P, Heikkilä P, Taipale J, Joensuu H, Rehn M, Pihlajaniemi T, Weich H, de Waal R and Alitalo K: VEGFR-3 and its ligand VEGF-C are associated with angiogenesis in breast cancer. Am J Pathol 154: 1381-1390, 1999.

30. Paavonen K, Puolakkainen P, Jussila L, Jahkola T and Alitalo K: Vascular endothelial growth factor receptor-3 in lymphangiogenesis in wound healing. Am J Pathol 156: 1499-1504, 2000 . 